\title{
Interaction of Nonhomogeneous Shear, Nonlinear Viscoelasticity, and Yield of a Solid Polymer
}

\author{
A. S. WINEMAN and W. K. WALDRON, JR. \\ Department of Mechanical Engineering \\ and Applied Mechanics \\ The University of Michigan \\ Ann Arbor, Michigan 48109
}

\begin{abstract}
A constitutive equation for small strain viscoelastic response is considered in which stress relaxation occurs faster as strain increases. The constitutive equation is of single integral type and has a psuedo- or material time function which is calculated from a strain dependent shift function. First, it is shown that such a constitutive equation can account for yield as observed in polymers for a number of different stress and strain histories. Next, the constitutive equation is used in the analysis of the problem in which a hollow cylinder is fixed at its inner surface and a moment history is applied to its outer surface. This causes the cylindrical surfaces to rotate about the central axis, thereby inducing a radial shear strain distribution. It is shown that there is a time when the materlal near the inner support begins to yield and a layer of large shear strain gradient begins to grow rapidly. It is also shown that the stress or strain history at a material element will generally not be one of the standard histories used to study yield.
\end{abstract}

\section{INTRODUCTION}

$\mathrm{T}$ There appear to be two distinct directions in the development of a constitutive equation which can model yield in polymers. In the first, the constitutive theory which has been developed for metal plasticity is applied directly to polymers. The second is to use the constitutive framework for nonlinear viscoelasticity. This approach has been the subject of a number of articles by Shay and Carruthers (1-4). They used a constitutive equation with certain mathematical features in carrying out a numerical simulation of constant extension rate experiments on polymer rods. Their results show the occurrence of the yield phenomenon. While their calculations are only in qualitative agreement with experimental results, it is clear that the constitutive equation, with appropriate modifications, is capable of an accurate simulation of yield. Knauss and Emri $(5,6)$, have discussed a constitutive equation for nonlinear viscoelasticity with similar features. Their calculations also indicate that yield occurs, although this was not the main purpose of their work.

There are additional reasons why a constitutive equation for nonlinear viscoelasticity is used to model yield in polymers. First, polymers exhibit creep and stress relaxation, and models of metal plasticity do not include this effect. Secondly, yield has been observed to occur under several different stress and strain histories (cf. Refs. 7-9). Since constitutive equations for viscoelasticity relate stress and strain histories, they are an appropriate framework for studies on yield. Thirdly, yield has been associated with a reduction of relaxation times with increasingly large deformation, an intrinsically viscoelastic effect (cf. Robertson (10)).

In this study, a constitutive equation for nonlinear viscoelasticity of the form used by Shay and Caruthers (1-4), and Knauss and Emri $(5,6)$, is given additional consideration. In their work, it is shown that yleld can be simulated under constant strain rate conditions. One purpose here is to show that the constitutive equation can also account for yield under the stress and strain histories considered in (8) and (9).

The above mentioned work involves only homogeneous deformations and specific stress or strain histories. A second purpose is to study a situation in which the stresses and strains vary in space as well as in time. The particular problem under consideration represents a potential experiment in which a hollow circular cylinder of a polymer is bonded to a rigid support at its inner surface and a rigid shell at its outer surface. The shell may undergo a prescribed rotation history or it may rotate due to a prescribed moment history. This induces, at each material element of the cylinder, the histories of a single shear stress component and the corresponding shear strain 
component. It is shown that these histories differ, in general, from the standard ones which have been used to define yield. However, yield-like phenomena can be defined and regions of yield in the cylinder can be identified.

The constitutive equation for nonlinear viscoelas ticity is presented in the section entitled Constitutive Equation. In the section on Yield, it is shown that this constitutive equation can be used to simulate yield under the various conditions which have appeared in the literature. Circumferential Shear of a Cylinder presents the geometry and governing equations. Finally, in Numerical Example, the method of solution is discussed and results are presented for two loading conditions, rotational control and moment control of the outer shell.

\section{CONSTITUTIVE EgUATION}

This work is concerned with conditions of geometric linearity (small strains and rotations) and material nonlinearity. In addition, attention is restricted to cases in which a stress component, $\sigma(t)$, is expressed only in terms of the corresponding strain component history $\epsilon(s), 0 \leq s \leq t$. The constitutive equation under consideration has the form

$$
\sigma(t)=\epsilon(0) G[\xi(t)]+\int_{0}^{t} G[\xi(t)-\xi(s)] \frac{d}{d s} \epsilon(s) d s
$$

Here $G(t)$ denotes a relaxation function associated with linear viscoelastic response, and $\xi(s)$ is a timelike variable which is affected by the strain history. It is given by the relation

$$
\xi(s)=\int_{0}^{s} \frac{d x}{\phi(\epsilon(x))},
$$

in which $\phi(\epsilon)$ is positive, and decreases monotonically with increasing strain.

This constitutive equation is a one-dimensional version of that used by Shay and Caruthers, (1), or Knauss and Emri, (5). In both papers, the authors began with a tensorial version of Eqn 1 which is appropriate for the three dimensional response of isotropic materials, and then applied it to uniaxial extension. This led to two equations in which the normal stress in the direction of extension and the normal stresses on transverse planes are related to the axial and transverse normal strains. These equations come from the condition in uniaxial extension that the two transverse normal strains are equal, due to material isotropy, and the normal stresses on transverse surfaces vanish. In concept, elimination of the transverse strain reduces this system to a single equation of form $E q$, although in practice, the equations must be solved simultaneously.

As will be shown in the next section, the mathematical property which causes Eqs 1 and 2 to account for yield is that $\phi$ decreases by several orders of magnitude as strain increases in some finite inter- val. This causes $\xi(t)$ to increase faster than time $t$ so that stress relaxation is accelerated as strain increases. The specific form used for $\phi$ in Refs. 1 and 5 was

$$
\log _{10} \phi=\frac{B}{2.303}\left(\frac{1}{f}-\frac{1}{f_{o}}\right)
$$

where $B$ is a material constant, $f$ is interpreted as the fractional free volume, and $f_{o}$ is a reference value of $f$. In both Refs. 1 and 5, the value of $f$ at any instant was calculated using the volumetric strain at that instant. The manner in which $f$ was calculated from the volumetric strain differed in these two papers. However, in both approaches, $f$ increased as elongation increased, resulting in a decrease in $\phi$.

Polymers also exhibit yield in shear. Experimentally determined shear stress-shear strain curves, obtained under conditions of constant shear strain rate, were presented by Bowden (7) and G'Sell and Gopez (11). These curves have the same characteristic shape as is used to define the occurrence of yield in uniaxial extensional response, namely a local maximum followed by a drop in the stress, followed by a rise in the curve. Both Bowden (7) and G'Sell and Gopez (11), refer to this as yield in shear. Since there must be some constitutive equation which can be used to simulate a yield experiment in shear, and since Eqs 1 and 2 can be shown to describe yield, as will be shown in the following section, the constitutive equation for shear will be assumed to have this same form for some appropriate choice of $\phi$ in $E q 2$. Then, $\sigma(t)$ and $\epsilon(s), 0 \leq s \leq t$, will represent the shear stress and the corresponding shear strain history, respectively.

In Refs. 1 and 5, yield in uniaxial extension was attributed to the acceleration of stress relaxation due to the increase in fractional free volume with increasing physical volume. The yield experiments of G'Sell and Gopez (11) are simple shear experiments in which there is no volume change. According to the constitutive equation in (1) and (2), stress relaxation will be accelerated due to shear deformations rather than volume changes. It is not intended here to relate this to a specific macromolecular theory, but instead to take a strictly phenomenological approach. A precedent for this is found in the work of McKenna and Zapas (12). They performed torsion experiments on PMMA rods in order to study its shear stress and normal stress response. Their data was analyzed in terms of the BKZ constitutive equation for nonlinear viscoelastic response. McKenna and Zapas showed that by allowing the time variable in their model, which is analogous to $\xi(s)$ in $E q 2$, to depend on the shear strain history, they could describe the response of mechanically conditioned PMMA. Thus, constitutive equations, Eqs 1 and 2, are chosen because they can simulate, at least in a qualitative sense, the phenomenon of yield in shear. When used along with the appropriate equations of mechanics to determine the stresses and strains in a sheared cylin- 
der, the results should provide insight into processes involving spatial variation of deformation and yield in shear.

This section is closed with the following comments pertaining to the use of Eqs 1 and 2 for shear response. It is expected that a relation between shear stress and shear strain is obtained as a component of a general constitutive equation for three-dimensional response. It is possible to develop a tensorial version of Eqs 1 and 2 which can be applied to the threedimensional response of isotropic materials, and in which $\phi$ depends on all the components of the strain tensor. For uniaxial response, this general form would give the relation between normal stresses and strains used in Refs. 1 or 5 , in which $\phi$ can depend on the volumetric strain. In shear, the general form reduces to Eqs 1 and 2 in which $\phi$ depends on the shear strain.

\section{YIELD}

Consider the uniaxial extension of a bar made of a solid polymeric material. The standard experiment for determining yield is one of strain control in which the specimen undergoes a constant strain rate history and the corresponding stress-time response is measured. Since the strain history is described by $\epsilon(t)=$ $\alpha t, t \geq 0$ where $\alpha$ is a constant, the time axis is re-scaled to a strain axis, and the stress-time graph is converted into a stress-strain graph. This is generally interpreted as the stress-strain curve for the material. Yield is said to occur if there is a point on the stress-strain curve where the stress has a local maximum. Note that the local maximum associated with yield is actually on the stress-time graph.

After reaching its value at yield, the strain increases while the stress first decreases slightly and then begins to increase. At some strain $\epsilon^{*}$ after the local maximum, there appears to be a change in the material microstructure, (7). If the stress is reduced to zero before $\epsilon^{*}$ is reached, the specimen will eventually return to its original length and there will be no permanent strain. Otherwise, there will be permanent strain, see Bowden (7), Fig. 5. The constitutive equations for viscoelastic solids which have been discussed in Refs. 1 and 5 are such that if stress is applied for some time and then maintained at zero for a sufficiently long time, the strains will reduce to zero. These constitutive equations are thus valid only for the regime near the local maximum.

The simulations in Refs. 1 and 5 describe the response to constant strain rate tests. However, yield has been defined to occur under other strain control conditions. Yee and DeTorres (9) carried out experiments in which the elongation increased first at one constant rate and then was changed to increase at a second constant rate. Results were obtained for various choices of the second elongation rate, both larger and smaller than the initial rate. The measured force-time graphs were transformed into forceelongation graphs by using the one to one relation between elongation and time. The force-elongation graphs had local maxima, indicating yield, and the yield points depended on the choice of elongation rates. It can be expected, based on these results, that local maxima, or yield, can occur for different monotonically increasing strain histories, and can depend on the particular history.

Yield has also been discussed under stress control conditions. Bowden (7) described a yield point when a specimen is subjected to a constant stress rate test. Carapellucci and Yee (8) conducted biaxial deformation experiments in which the stress rates were constant and the strain-time responses were measured. The strain increased monotonically and approached a very large rate of growth at some finite time. In other terms, the strain-time curve had a vertical asymptote. This rapid growth of strain is defined to be yield. The stress control history is described by $\sigma(t)=\beta t$, $t \geq 0$ where $\beta$ is constant. This can be used to transform the time axis into a stress axis thereby creating another stress-strain graph. The vertical asymptote on the strain-time graph becomes a horizontal asymptote at some finite stress on the stress-strain graph, which is an indicator of yield.

The preceding discussion points out that the definition of yield depends on the particular stress or strain history that is being controlled. Although yield is usually defined in terms of a feature of a stress-strain curve, this feature is first observed on a stress-time or strain-time graph. It will now be shown that the proposed constitutive equation can simulate yield under these different stress and strain histories.

Let $E q 1$ be differentiated with respect to time and use $E q 2$. The result is

$$
\begin{aligned}
\dot{\sigma}(t)= & \epsilon(0) \frac{\dot{G}[\xi(t)]}{\phi(\epsilon(t))}+G(0) \dot{\epsilon}(t) \\
& +\frac{1}{\phi(\epsilon(t))} \int_{0}^{t} \dot{G}[\xi(t)-\xi(s)] \dot{\epsilon}(s) d s,
\end{aligned}
$$

where the superposed dot denotes the derivative of the function with respect to its argument.

Now consider a constant strain rate history, $\epsilon(t)=$ $\alpha t, t \geq 0$ where $\alpha$ is constant. Equation 4 becomes

$$
\dot{\sigma}(t)=\alpha\left[G(0)+\frac{1}{\phi(\alpha t)} \int_{0}^{t} \dot{G}[\xi(t)-\xi(s)] d s\right] .
$$

The relaxation function has the property that $G(s) \geq$ $0, \dot{G}(s) \leq 0, s \geq 0$. Thus, the first term in $E q 5$ is positive and the integral is negative (recall that $\phi(\epsilon)$ is also positive). As the strain increases, $\phi(\alpha t)$ decreases by several orders of magnitude, and can become extremely small. At some time $t^{*}$, the right hand side of $E q 5$ vanishes,

$$
G(0)+\frac{1}{\phi\left(\alpha t^{*}\right)} \int_{0}^{t^{*}} \dot{G}\left[\xi\left(t^{*}\right)-\xi(s)\right] d s=0,
$$

and the stress-time graph will have a local maximum. When the time axis is re-scaled by the relation $\epsilon(t)=$ $\alpha t$, the result is a stress-strain curve with the local 


\section{A. S. Wineman and W. K. Waldron, Jr.}

maximum which characterizes yield. This is the basis for the yield curves in Refs. 1 and 5 .

Now consider a continuous strain history which increases monotonically from the undeformed state. Then $\epsilon(0)=0$ and $\dot{\epsilon}(t) \geq 0, t \geq 0$. The leading term in $E q 4$ vanishes, the second term is positive and the integral is negative. As the strain increases, $\phi(\epsilon(t))$ decreases monotonically to a value sufficiently small that at some time $\hat{t}, \dot{\sigma}(\hat{t})=0$. The strain histories of Yee and DeTorres (9) were the special case in which $\dot{\epsilon}(t)=\alpha_{1}, 0 \leq t<T_{o}$ and $\dot{\epsilon}(t)=\alpha_{2}, t \geq T_{o}$, where $\alpha_{1}$, $\alpha_{2}$, and $T_{o}$ are constants.

Finally, consider the constant stress rate test, $\sigma(t)$ $=\beta t, t \geq 0$ where $\beta$ is positive. Since $\sigma(0)=0$ implies $\epsilon(0)=0$, the strain rate $\dot{\epsilon}(t)$ is found from $E q 4$ to be given by the relation

$$
\dot{\epsilon}(t)=\frac{1}{G(0)}\left[\beta-\frac{1}{\phi(\epsilon(t))} \int_{0}^{t} \dot{G}[\xi(t)-\xi(s)] \dot{\epsilon}(s) d s\right] .
$$

According to $E q 7, \dot{\epsilon}(0)>0$ so that $\dot{\epsilon}(s)>0$ in some time interval. The right hand side of $E q 7$ is seen to be positive, which implies that $\dot{\epsilon}(t)$ is always positive. Thus $\epsilon(t)$ increases monotonically, $\phi(\epsilon(t))$ decreases and again becomes extremely small. Correspondingly, $\dot{\boldsymbol{\epsilon}}(t)$ becomes very large in some finite time interval and the material is said to be undergoing yield. This describes the experimental results of Carapellucci and Yee (8). Finally, consider general stress histories for which $\sigma(0)=0$ and $\dot{\sigma}(t) \geq 0, t \geq 0$. A similar discussion again leads to the conclusion that $\dot{\epsilon}(t)$ becomes very large in some finite time interval.

Although yield is normally defined for specific strain and stress histories, the above discussion makes it clear that yield can also occur for many other stress or strain histories. This will be illustrated using the example presented in Circumferential Shear of a Cylinder and Numerical Example sections.

\section{CIRCUMFERENTIAL SHEAR OF A CYLINDER}

Consider a hollow circular cylinder made of a solid polymer. Its inner and outer radii are $R_{i}$ and $R_{o}$, respectively, and its length is $L$. The inner surface is bonded to a rigid cylindrical support and the outer surface is bonded to a rigid shell. A time dependent moment is applied to the outer shell which causes it to undergo a time dependent rotation. The resulting relative rotation between the inner and outer surfaces of the cylinder induces a non-homogeneous shear stress and shear strain distribution at each instant. These are to be determined when the material has the constitutive equation discussed in the sections on Constitutive Equation and Yield, as is the relation between the rotation of the cylinder and the applied moment.

It is assumed that the cylinder is sufficiently long that end effects and axial variation of quantities can be neglected. The deformation is assumed to be axi- ally symmetric, quasi-static, and sufficiently small that the small strain approximation is valid.

Let $u_{r}, u_{\theta}, u_{z}$ denote displacement components with respect to a cylindrical coordinate system. The assumptions imply that

$$
u_{r}=u_{z}=0, u_{\theta}=u(r, t)
$$

The only non-vanishing component of the infinitesimal strain tensor is $\epsilon_{r \theta}$. Let the corresponding geometric shear strain be denoted by $\gamma_{r \theta}$. Then $\gamma_{r \theta}=2 \epsilon_{r \theta}$ and it is related to $u(r, t)$ by

$$
\gamma_{r \theta}=r \frac{\partial}{\partial r}\left(\frac{u}{r}\right)
$$

or

$$
\gamma_{r \theta}=r \frac{\partial}{\partial r}(\omega(r, t))
$$

where $\omega(r, t)=u(r, t) / r$ is the rotation of the cylindrical surface of radius $r$ at time $t$.

The cylinder material is assumed to be an isotropic nonlinear viscoelastic solid. The general form of the constitutive equation ( $E q 1)$ for three dimensional stress and strain rates, restricted for geometric linearity, is presented in Refs. 1 and 5. Since at each material element of the cylinder the only non-zero strain is $\gamma_{r \theta}$, it is found from the general constitutive eqquation that the only non-zero stress is $\sigma_{r \theta}$. These are related by $E q 1$ which is restated in terms of the present variables,

$$
\sigma_{r \theta}(t)=\gamma_{r \theta}(0) G[\xi(t)]+\int_{0}^{t} G[\xi(t)-\xi(s)] \dot{\gamma}_{r \theta}(s) d s
$$

where $\xi(s)$ is given by $E q 2$ in which $\epsilon$ is replaced by $\gamma_{r \theta}$.

Since the deformation is quasi-static, the stress must satisfy equilibrium conditions at each time $t$. It can be shown by using either the differential equations of equilibrium or the equation for the balance of moments for a portion of the cylinder between a typical radius $r$ and the outer surface, that

$$
\sigma_{r \theta}(r, t)=\frac{M(t)}{2 \pi r^{2}}
$$

Here $M(t)$ is the moment per unit length at time $t$ which is applied to the outer shell.

Let Eqs 11 and 12 be combined to give

$$
\begin{aligned}
\frac{M(t)}{2 \pi r^{2}}= & \gamma_{r \theta}(r, 0) G[\xi(r, t)] \\
& +\int_{0}^{t} G[\xi(r, t)-\xi(r, s)] \frac{\partial \gamma_{r \theta}(r, s)}{\partial s} d s,
\end{aligned}
$$

where, by $E q 2$,

$$
\xi(r, s)=\int_{0}^{s} \frac{d x}{\phi\left(\gamma_{r \theta}(r, x)\right)}
$$


These represent equations for $\gamma_{r \theta}(r, t)$. Once this has been determined, $\omega(r, t)$ can easily be obtained from $E q 10$ using the boundary condition at the fixed support that $\omega\left(R_{i}, t\right)=0$. The rotation of the outer cylinder is given by $\omega\left(R_{o}, t\right)$.

Two different loading conditions will be considered: Moment Control-in which the moment history $M(t)$ is specified, and Rotation Control-in which the rotation history $\omega\left(R_{o}, t\right)$ is specified. For each of these problems, it will be possible to determine the strain and rotation at each time, and to construct the stress-strain relation at each particle and the $M-\omega$ relation for the cylinder.

For the purposes of later discussion, it will be useful to determine the solution when the material is isotropic and linearly viscoelastic. This is characterized by the condition that $\phi=1$ in $E q 14$, so that $\xi(t)=t$, i.e. independent of strain. Equation 11 reduces to the constitutive equation for linear viscoelasticity,

$$
\sigma_{r \theta}(t)=\gamma_{r \theta}(0) G(t)+\int_{0}^{t} G(t-s) \dot{\gamma}_{r \theta}(s) d s
$$

This equation can be inverted to give the strain history in terms of the stress history

$$
\gamma_{r \theta}(t)=\sigma_{r \theta}(0) J(t)+\int_{0}^{t} J(t-s) \dot{\sigma}_{r \theta}(s) d s
$$

where $J(t)$ denotes the creep function of the material. Using the system consisting of Eqs 10,12, 15, and 16 , the rotation and moment control problems can be solved in a quite straightforward manner. The details of their solution will be omitted, and only the final results will be presented here. Attention will be restricted to control histories which increase at a constant rate.

\section{Moment Control}

Consider the moment history $M(t)=\dot{M}_{o} t$, where $\dot{M}_{o}$ is a constant. It follows from $E q 12$ that the stress is given by

$$
\sigma_{r \theta}(r, t)=\frac{\dot{M}_{o}}{2 \pi r^{2}} t
$$

This shows that each material element experiences a constant stress rate history, but that the rate varies with radius. The corresponding strain history is found to be given by

$$
\gamma_{r \theta}(t)=\frac{\dot{M}_{o}}{2 \pi r^{2}} \int_{0}^{t} J(x) d x .
$$

A shear stress-shear strain relation can be constructed for each particle by eliminating time between Eqs 17 and 18. This can be written in the form

$$
\gamma_{r \theta} / \dot{\sigma}_{o}=\int_{0}^{\sigma_{r \theta} / \dot{\sigma}_{o}} J(x) d x
$$

where

$$
\dot{\sigma}_{o}=\frac{\dot{M}_{o}}{2 \pi r^{2}}
$$

The rotation history $\omega\left(R_{o}, t\right)$ is

$$
\omega\left(R_{o}, t\right)=\frac{R_{o}^{2}-R_{i}^{2}}{R_{o}^{2} R_{i}^{2}} \frac{\dot{M}_{o}}{4 \pi} \int_{0}^{t} J(x) d x,
$$

which implies the moment-rotation relation

$$
\omega=\frac{R_{o}^{2}-R_{i}^{2}}{R_{o}^{2} R_{i}^{2}} \frac{\dot{M}_{o}}{4 \pi} \int_{0}^{M / \dot{M}_{o}} J(x) d x
$$

\section{Rotation Control}

Consider the rotation history $\omega\left(R_{o}, t\right)=\dot{\omega}_{o} t$, where $\dot{\omega}_{o}$ is a constant. The strain $\gamma_{r \theta}$ is found to be

$$
\gamma_{r \theta}(r, t)=\frac{2 R_{i}^{2} R_{o}^{2}}{R_{o}^{2}-R_{i}^{2}} \frac{\dot{\omega}_{o}}{r^{2}} t .
$$

Note that each material element undergoes a constant rate strain history, but the strain rate varies with radius. The stress is given by

$$
\sigma_{r \theta}(r, t)=\frac{2 R_{i}^{2} R_{o}^{2}}{R_{o}^{2}-R_{i}^{2}} \frac{\dot{\omega}_{o}}{r^{2}} \int_{0}^{t} G(x) d x,
$$

and the moment history by

$$
M(t)=\frac{R_{i}^{2} R_{o}^{2}}{R_{o}^{2}-R_{i}^{2}} 4 \pi \dot{\omega}_{o} \int_{0}^{t} G(x) d x .
$$

A shear stress-shear strain relation follows from $E q s$ 22 and 23 , and can be written as

$$
\sigma_{\mathrm{r} \theta} / \dot{\gamma}_{o}=\int_{0}^{\gamma_{r \theta} / \dot{\gamma}_{o}} G(x) d x
$$

in which

$$
\dot{\gamma}_{o}=\frac{2 R_{i}^{2} R_{o}^{2}}{R_{o}^{2}-R_{i}^{2}} \frac{\dot{\omega}_{o}}{r^{2}} .
$$

Finally, the moment-rotation relation is found, using $E q 24$ to be given by

$$
M=\frac{R_{i}^{2} R_{o}^{2}}{R_{o}^{2}-R_{i}^{2}} 4 \pi \dot{\omega}_{o} \int_{0}^{\omega / \dot{\omega}_{o}} G(x) d x .
$$

\section{NUMERICAL EXAMPLE}

It does not appear possible to obtain a general solution to Eqs 13 and 14 for nonlinear response, analogous to that presented in Eqs 17-26 for linear response. Instead, a numerical solution is presented. For notational convenience let $\gamma=\gamma_{r \theta}$. The functions $G(t)$ and $\phi(\gamma)$ are chosen, for the purpose of this numerical example, to represent properties of a typical material, rather than a specific material. The shear relaxation function associated with linear response is taken to have the form

$$
G(t)=G_{o}\left[(1-g) e^{-t / \tau_{o}}+g\right]
$$


in which $G_{o}=G(0), g=G(\infty) / G(0)$ and $\tau_{o}$ is a characteristic relaxation time. Let $\hat{G}(t, \gamma)$ denote a shear strain dependent stress relaxation function. Its form is assumed to be

$$
\hat{G}(t, \gamma)=G_{o}\left[(1-g) e^{-t / \tau(\gamma)}+g\right],
$$

in which $\tau(\gamma)$ is a shear strain dependent characteristic relaxation time. Define

$$
\phi(\gamma)=\frac{\tau(\gamma)}{\tau_{o}}
$$

where $\tau_{o}=\tau(0)$. It follows from Eqs 27, 28, and 29 that

$$
\hat{G}(t, \gamma)=G(t / \phi(\gamma)),
$$

and hence that $\phi(\gamma)$ can be interpreted as the shear strain dependent shift function in Eqs 2 and 14.

A form for $\phi(\gamma)$ is based on the assumption that $\tau(\gamma)$ is given by a relation of the form

$$
\tau(\gamma)=a e^{\frac{B}{p(\gamma)}}
$$

in which $p(\gamma)$ is a positive, monotonically increasing function of $|\gamma|$. Then $\tau(\gamma)$ decreases as $\gamma$ increases. This relation is chosen to be similar in form to the relaxation time-temperature relation in the statistical thermodynamical theory of Adam and Gibbs which is presented by Ward in Ref. 13.

It follows from $E q 29$ that

$$
\log _{10} \phi(\gamma)=\frac{B}{2.303}\left[\frac{1}{p(\gamma)}-\frac{1}{p(0)}\right] \text {. }
$$

In the present work

$$
p(\gamma)=p_{o}+\theta|\gamma| \text {. }
$$

When $\theta=0, \phi(\gamma)=1$ and the constitutive equation reduces to that for linear viscoelasticity. In the computational results presented here, $\theta=0.05$. The values of the parameters in Eqs 32 and 33 are $B=0.474$ and $p_{o}=0.0217$, and are the same as used for the PVAc in calculations by Knauss and Emri (5). Let the following non-dimensionalization be defined:

$$
\begin{aligned}
\bar{s}=s / \tau_{o}, & \bar{t}=t / \tau_{o}, \\
\bar{r}=r / R_{i}, & \bar{R}_{o}=R_{o} / R_{i}, \\
\bar{\xi}(\bar{r}, \bar{s})=\xi(r, s) / \tau_{o}, & \bar{\xi}(\bar{r}, \bar{t})=\xi(r, t) / \tau_{o}, \\
\bar{\gamma}(\bar{r}, \bar{t})=\gamma(r, t), & \bar{\omega}(\bar{r}, \bar{t})=\omega(r, t), \\
\bar{G}(\bar{t})=G(t) / G_{o}, & \bar{M}(\bar{t})=\frac{M(t)}{2 \pi G_{o} R_{i}^{2}}
\end{aligned}
$$

The non-dimensional forms of Eqs 13 and 14 are

$$
\begin{gathered}
\frac{\bar{M}(\bar{t})}{\bar{r}^{2}}=\bar{\gamma}(\bar{r}, \bar{t})+\int_{0}^{t} \frac{\dot{\bar{G}}[\bar{\xi}(\bar{r}, \bar{t})-\bar{\xi}(\bar{r}, \bar{s})]}{\phi[\bar{\gamma}(\bar{r}, \bar{s})]} \bar{\gamma}(\bar{r}, \bar{s}) d \overline{\mathbf{s}} \\
\bar{\xi}(\bar{r}, \overline{\mathbf{s}})=\int_{0}^{\bar{s}} \frac{d \bar{x}}{\phi(\bar{\gamma}(\bar{r}, \bar{x}))}
\end{gathered}
$$

Let a moment history be specified. For each fixed radius, Eqs 35 and 36 define a nonlinear Volterra integral equation for the shear strain history at the radius. The numerical solution is based on methods presented by Linz (14) and has been used in earlier work, e.g. Wineman (15). At radial node $\bar{r}_{i}$ in the interval $\left[1, \bar{R}_{o}\right]$, the shear strain is obtained at a discrete set of times $\bar{t}_{1}=0, \bar{t}_{2}, \ldots, \bar{t}_{n}$ as follows. At time $\bar{t}_{n}$, the integrals in Eqs 35 and 36 are approximated by a finite summation which is expressed in terms of $\bar{\gamma}\left(\bar{r}_{i}, \bar{t}_{j}\right), j=1, \ldots, n$. If $\bar{\gamma}\left(\bar{r}_{i}, \bar{t}_{j}\right)$ have been found for $j=1, \ldots, n-1$, then Eqs 35 and 36 become an equation for $\bar{\gamma}\left(\bar{r}_{i}, \bar{t}_{n}\right)$. This equation is solved by iteration. When the solution has been found at time $\bar{t}_{n}$, the process is repeated at time $n+1$. The radial distribution of rotations of cylindrical surfaces at time $\bar{t}_{n}$ is obtained by numerical integration of $E q$ 10. Further details are omitted here for the purpose of brevity. Calculations were carried out for the case in which $g=0.001$ and $\bar{R}_{o}=2$.

\section{Moment Control}

A constant moment rate history, $M(t)=\dot{M}_{o} t$ was selected so that results for the nonlinear case $(\theta=$ 0.05) could be compared with results for the linear viscoelastic case $(\theta=0)$ presented in Circumferential Shear of a Cylinder. Let this moment history be substituted into $E q 35$ and let the non-dimensionalization from $E q 34$ be introduced. The left hand side of Eq 35 becomes $\dot{\bar{M}}_{o} \bar{t} / \bar{r}^{2}$, where $\dot{\bar{M}}_{o}=M_{o} \tau_{o} / 2 \pi G_{o} R_{i}^{2}$. Calculations were carried out for $\dot{\bar{M}}_{o}=0.0001$.

According to $E q 17$, this moment history causes each material element to be subjected to a constant shear stress rate history, with the stress rate a maximum at the inner boundary and decreasing with radius. Each material element then has a monotonically increasing strain history. Figure 1 shows shear strain histories for material elements at the inner boundary $\bar{r}=1$, near the boundary, at $\bar{r}=1.2$, and at the outer boundary $\bar{r}=2$. Histories are shown for both the nonlinear case $(\theta=0.05)$ and the linear case $(\theta=0)$. Results are shown only for the initial time interval when $\gamma<0.08$, which is near the limit of validity of the small strain assumption. At each radius, the strains for $\theta=0.05$ increase faster than those for $\theta=0$. For sufficiently small times, the strain history for $\theta=0.05$ is very close to the strain history for $\theta=0$. When the shear strain increases to about 0.002 , the strain history for $\theta=0.05$ begins to increase noticeably faster than that for $\theta=0$. At $\bar{r}=1$, this occurs at about $\bar{t}=5$. At $\bar{r}=1.2$, it occurs at $\bar{t}=6$ while at $\bar{r}=2$, it does not occur until $\bar{t}=12$. The difference between the strain histories increases at both time and strain increase. Since the shear stress rate is greatest at the inner boundary, this is the region where the difference grows fastest. This effect is indicated in Fig. 2. The strain-time plot for $\bar{r}=1$, $\theta=0.05$, becomes very steep at $\bar{t}=20$, while the others are still gradually increasing.

The shear strain distributions at several times are shown in Fig. 2. In each case, the strain is a maxi- 


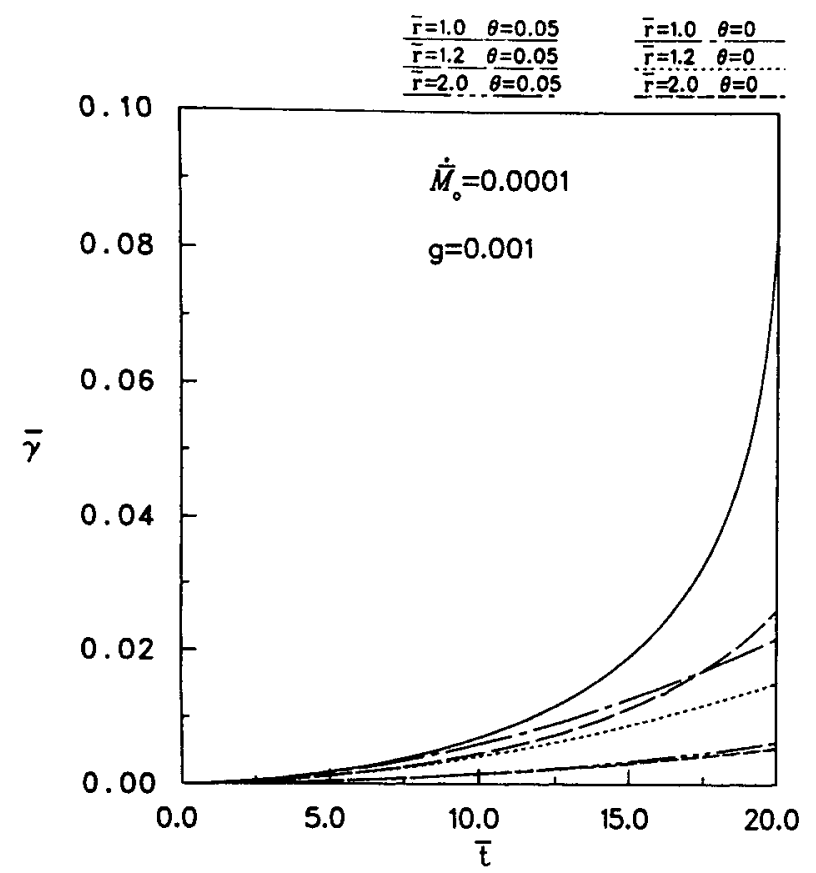

Fig. 1. Moment control: shear strain vs. time at radii $\bar{r}=1$, 1.2 , and 2.0 .

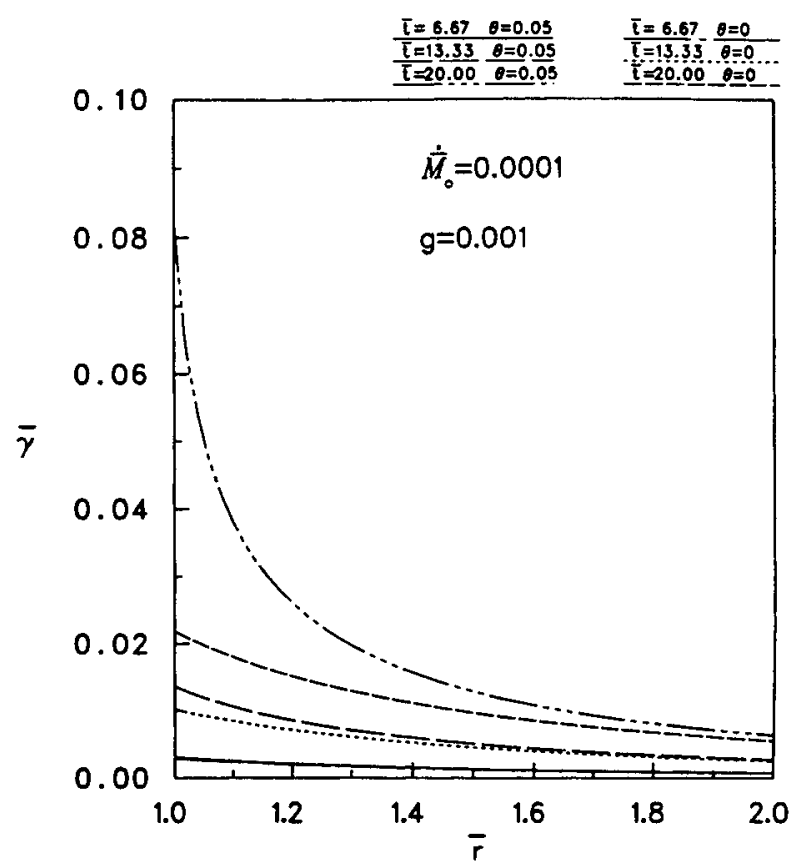

Fig. 2. Moment control: shear strain us. radius for times $\bar{t}=6.67,13.33$, and 20.0 .

mum at the inner boundary and decreases monotonically with increasing radius. This is a consequence of a similar radial distribution in shear stress rate. At $\bar{t}=6.67$, the maximum shear strain is still small and the strain distributions for $\theta=0.05$ and $\theta=0$ are very close. At $\bar{t}=13.33$, the difference in the distributions has increased, and is greatest near the inner boundary. As time increases to $t=20$, the difference in- creases much more rapidly near the inner boundary. A zone of large shear strain and very large strain gradient develops near the inner support.

Figures 3 and 4 provide useful insight into the mechanism of development of the zone of large shear strain. Figure 3 shows histories of $\bar{\xi}(\bar{r}, \bar{t})$ us. $\bar{t}$ for various radii and Fig. 4 shows $\bar{\xi}(\bar{r}, \bar{t})$ us. $\bar{r}$ for various times. When $\theta=0$, it follows from Eqs 32, 33, and 36 that $\bar{\xi}(\bar{r}, \bar{t})=\bar{t}$. In Fig. $3, \bar{\xi}$ us. $\bar{t}$ for $\theta=0$ corresponds to the continuation of the straight line near $\bar{t}=0$

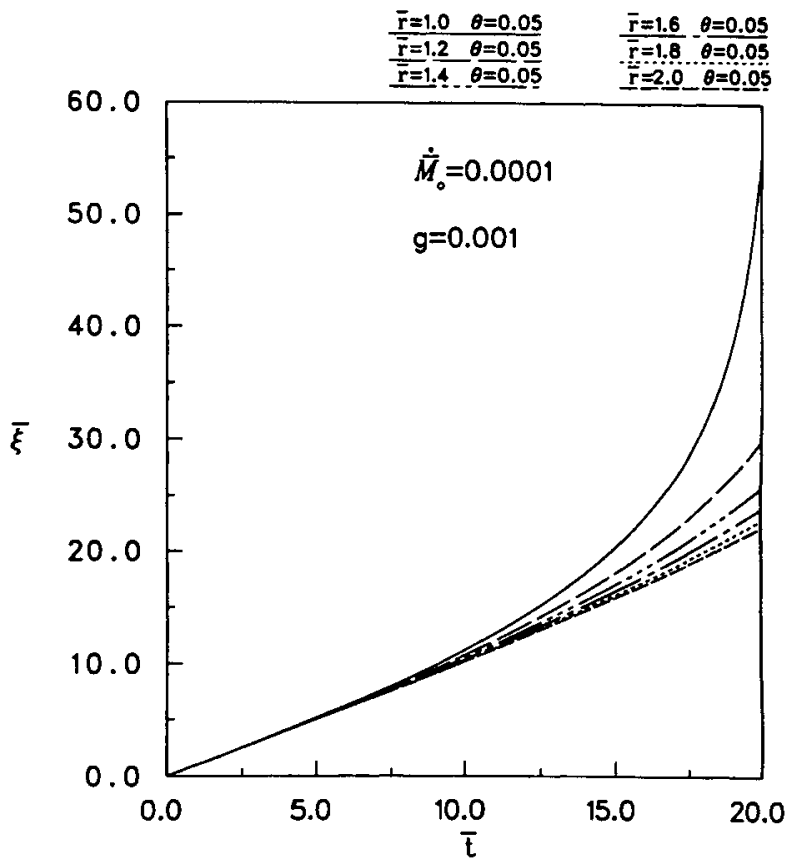

Fig. 3. Moment control: reduced time vs. time at several radii.

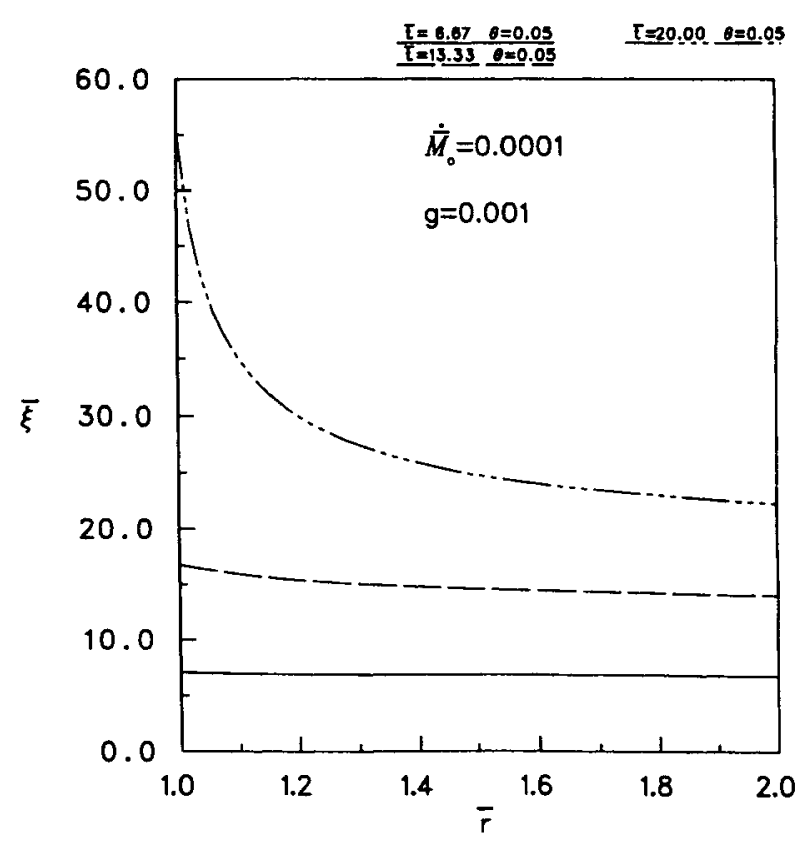

Fig. 4. Moment control: reduced time us. radius for times $\bar{t}=6.67,13.33$, and 20.0 . 
common to all histories. In Fig. $4, \bar{\xi}$ us. $\bar{r}$ for $\theta=0$ is a horizontal line. Figures 3 and 4 show that when $\theta=0.05, \bar{\xi}$ grows faster than $\bar{t}$, this growth being faster as $\bar{t}$ increases. It is fastest near $\bar{r}=1$, and decreases with the radius. Since $\bar{\xi}$ accounts for a strain dependent stress relaxation process, these results imply that when $\theta=0.05$, stress relaxation is faster than when $\theta=0$, is fastest near $\bar{r}=1$, and decreases rapidly with radius.

In other terms, as the shear strain increases, the shift function $\phi(\gamma)$ decreases, $\bar{\xi}$ increases faster than real time $\bar{t}$, and stress relaxation occurs faster. When the shear strain is small, the effect is minor and there is not much difference between the linear and nonlinear cases. When the strain becomes sufficiently large, the decrease in the shift function and the increase in the rate of stress relaxation are significantly accelerated. The difference between the linear and nonlinear cases then becomes substantial. In effect, the conditions for yield under constant stress rate, as discussed in the section on Yield, are being satisfied for particles in the neighborhood of the inner support.

The rotation histories of cylindrical surfaces at $\bar{r}=$ 1.2 and $\bar{r}=1.4$ near the inner boundary and at $\bar{r}=2$ near the outer boundary are shown in Fig. 5. The radial distributions of $\omega$ are shown in Fig. 6. The growth is quite gradual when $\theta=0$. However, for $\theta=0.05$, there is a rapid increase in rotation after $\bar{t}=15$. As seen from $E q 1 O$, this is due to the rapid increase in shear strain near $\bar{r}=1$.

Recalling that the moment history is $\bar{M}(\bar{t})=\dot{\bar{M}}_{o} \bar{t}$, the time axis in Fig. 5 can be re-scaled as a moment axis. This leads to a plot of the moment $v$ s. rotation of the outer radius shown in Fig. 7 .

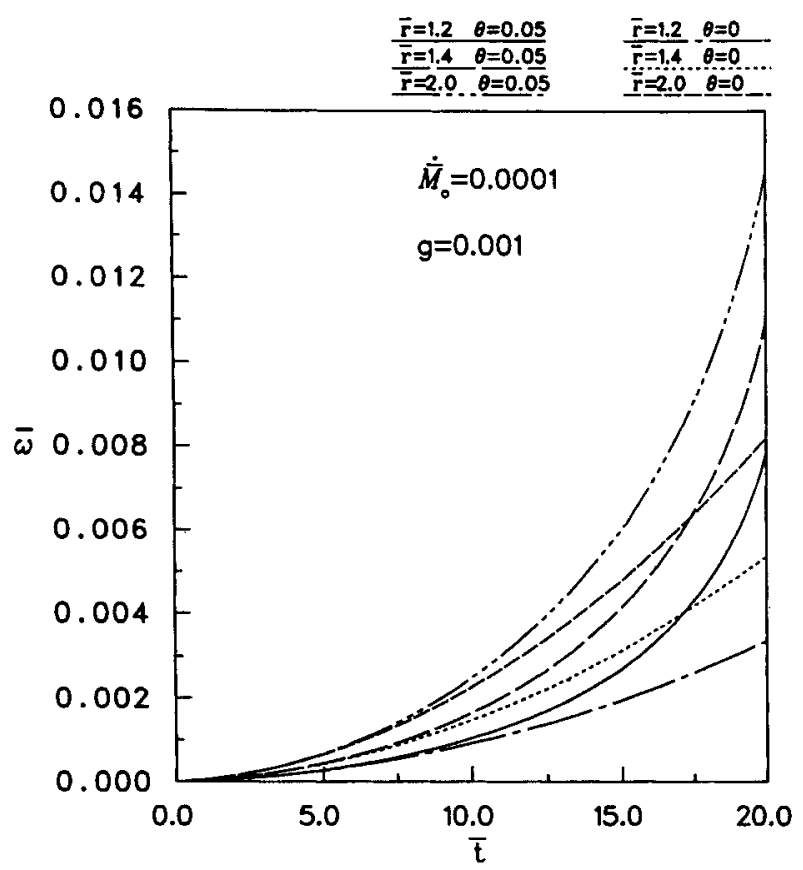

Fig. 5. Moment control: cylindrical surface rotation vs. time at radii $\vec{r}=1.2,1.4$, and 2.0 .

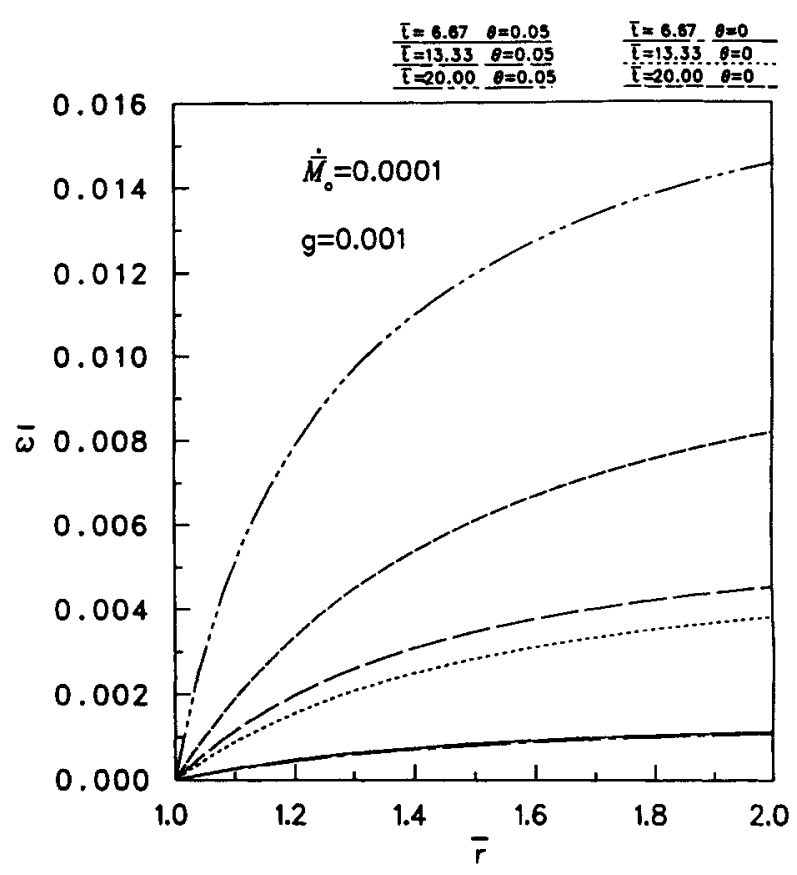

Fig. 6. Moment control: cylindrical surface rotation us, radius for times $\bar{t}=6.67,13.33$, and 20.0 .

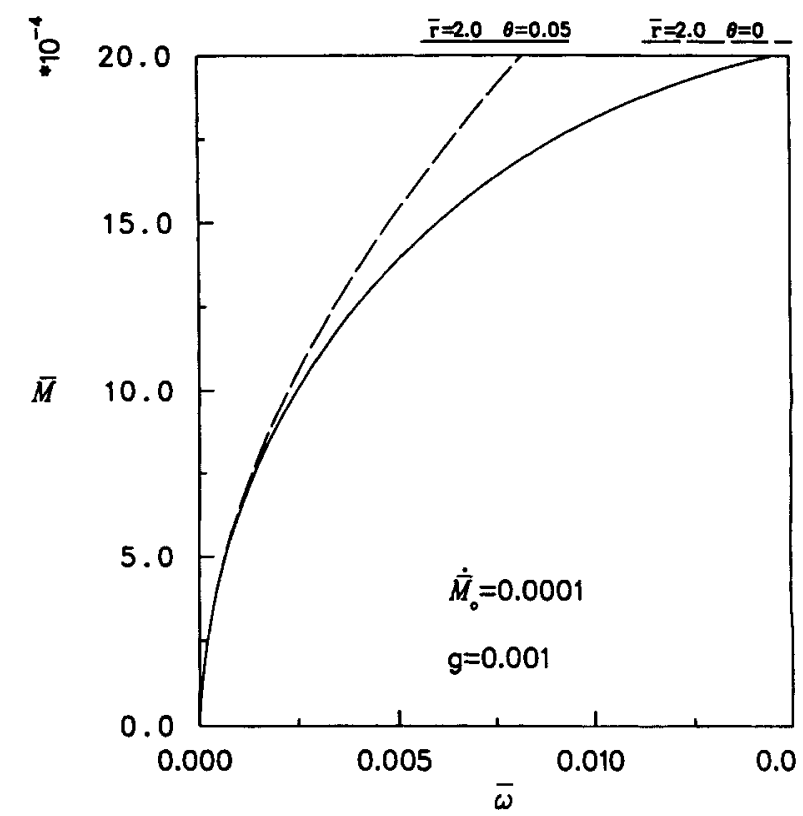

Fig. 7. Moment control: moment vs. rotation of outer surface.

Figure 8 shows shear stress us. shear strain for the material elements at $\bar{r}=1, \bar{r}=1.2$, and $\bar{r}=2$. Plots are shown for both $\theta=0$ and $\theta=0.05$. The plot corresponding to $\bar{r}=1, \theta=0.05$, has a large horizontal portion, characteristic of yield under constant stress rate conditions. The curves for $\bar{r}=1.2, \theta=0.05$, is just beginning to indicate yield. However, the strains at $\bar{r}=1.2$ never increase to a level suggesting yield has occurred. The figure thus points out an important feature of using a model for nonlinear viscoelasticity to account for yield. When the deformation 


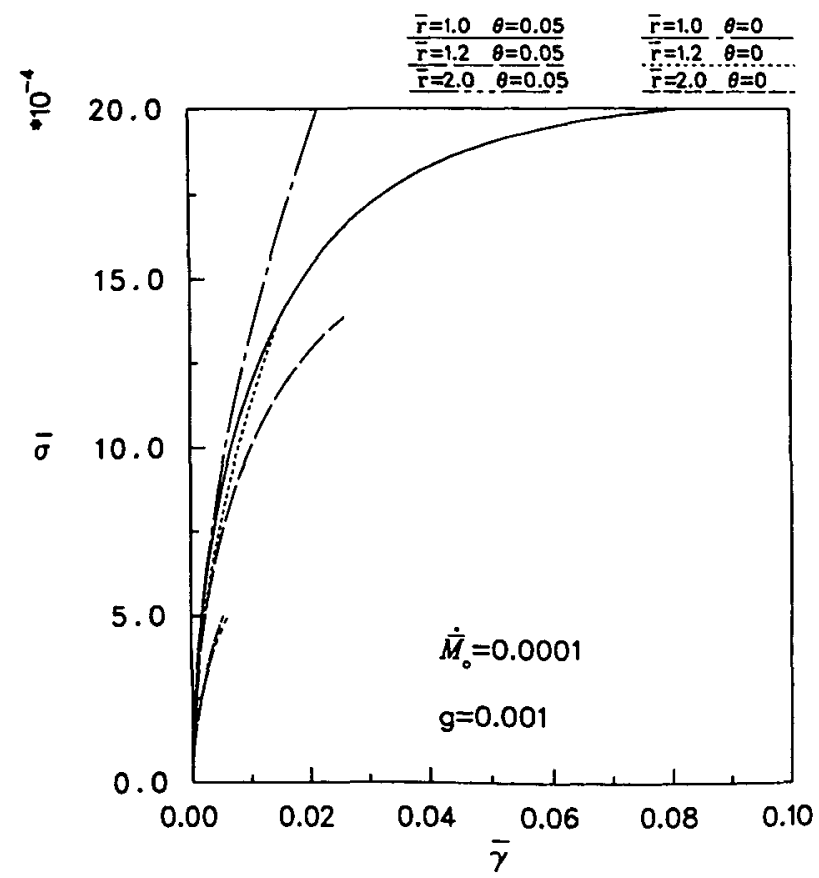

Fig. 8. Moment control: shear stress us, shear strain at radii $\bar{r}=1.0,1.2$, and 2.0 .

varies spatially, the model allows for a continuous transition to yield-like behavior. It eliminates the necessity of introducing a yield criterion, and the associated difficulty of determining where yield occurs.

Another comment should be made in conjunction with Fig. 8. The material elements of the cylinder are identical. Yet, for both $\theta=0$ and $\theta=0.05$, each material element has a different stress-strain plot. This is a consequence of the different strain history at each element. It is common practice in the literature $(1,5$, 7-9) to present a stress-strain plot for a material. This figure shows that a material element does not have a unique stress-strain plot. In applications, where conditions vary spatially, the concept of a stress-strain plot may be of little use.

\section{Rotation Control}

The outer cylinder was assumed to undergo a constant rotation rate history $\omega\left(R_{o}, t\right)=\dot{\omega}_{o} t$ or in dimensionless form $\bar{\omega}\left(\bar{R}_{o}, \bar{t}\right)=\dot{\bar{\omega}}_{\mathrm{o}} \bar{t}$, where $\dot{\bar{\omega}}_{o}=\dot{\omega}_{o} \tau_{\mathrm{o}}$. Results for the nonlinear case $(\theta=0.05)$ can then be compared with results for the linear case $(\theta=0)$, which has the analytical solution presented in the section on Circumferential Shear of a Cylinder. Calculations were carried out for $\dot{\bar{\omega}}_{o}=0.001$.

Rotation control requires a modification of the numerical solution outlined above. Suppose that $\bar{M}\left(\bar{t}_{j}\right)$ and $\bar{\gamma}\left(\bar{r}_{i}, \bar{t}_{j}\right)$ have been found, $j=1,2, \ldots, n-1$. At time $\bar{t}_{n}, \bar{M}\left(\bar{t}_{n}\right)$ is estimated and $\bar{\gamma}\left(\bar{r}_{i}, \bar{t}_{n}\right)$ is solved for at each radial node using Eqs 35 and 36 . Equation 10 is integrated numerically to obtain a value for $\bar{\omega}\left(\bar{R}_{o}, \bar{t}_{n}\right) . \bar{M}\left(\bar{t}_{n}\right)$ is then adjusted until $\mid \bar{\omega}\left(\bar{R}_{o}, \bar{t}_{n}\right)-$ $\dot{\bar{\omega}}_{\mathrm{o}} \bar{t}_{n} \mid$ is within a specified tolerance.
Before presenting the numerical results, it will be useful to discuss the analytical solution for the linear case $(\theta=0)$, in the previous section. It is seen from $E q 22$ that each material element undergoes a constant strain rate history, with the strain rate decreasing with increasing radius. At a fixed time, the shear strain decreases as $1 / \bar{r}^{2}$. It is easily found from Eqs 23 and 24 that at a fixed radius, the stress rate and moment rate are proportional to $G(t)$, and thus decrease monotonically with time. Near $t=0$, these rates are proportional to $G(0)$, and subsequently decrease to being proportional to $G(\infty)$.

The moment history required to impose the constant rotation rate history on the outer cylinder is shown in Fig. 9 for both $\theta=0$ and $\theta=0.05$. The $\bar{M} v$ s. $\bar{t}$ graph for $\theta=0$ is consistent with the analytical results discussed above. The $\bar{M}$ vs. $\bar{t}$ graph is monotonic, with a slope decreasing from a value proportional to $G(0)=1$ to a value proportional to $\bar{G}(\infty)=$ 0.001 and this appears to be nearly horizontal.

The solution for $\theta=0.05$ is completely different. The $\bar{M}$ us. $\bar{t}$ graph reaches a local maximum at about $\bar{t}=2.5$ and then begins to decrease. The reason for this can be seen in the shear strain histories in Fig. 10 and shear strain distributions in Fig. 11. Since the shear stress is greatest near the inner boundary, the shear strain is also greatest there. Hence, stress relaxes faster there. This in effect softens the response and allows the strain to increase faster. According to $\mathrm{Eq} 10$, this causes most of the contribution to the rotation of the outer cylinder to come from the shear distortion of the material near the inner support. In order to ensure that $\bar{\omega}\left(\bar{R}_{o}, \bar{t}\right)$ grows at the prescribed rate, the moment history must begin to decrease at some time. By Eq 12, the shear stress at each radius will be decreased, thereby slowing the

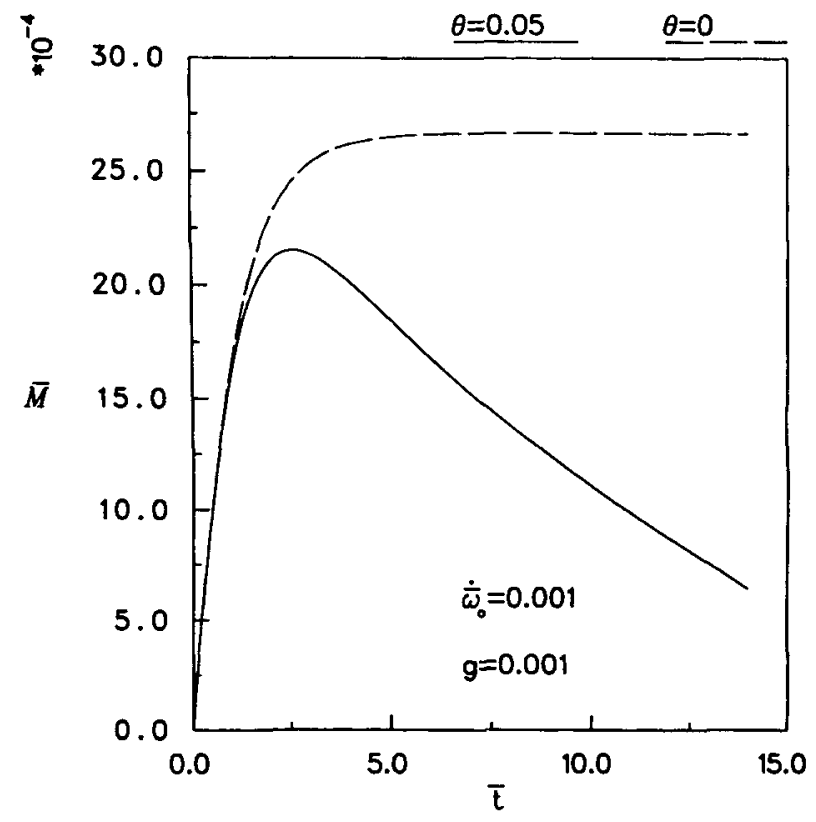

Fig. 9. Rotation control: moment us. time. 

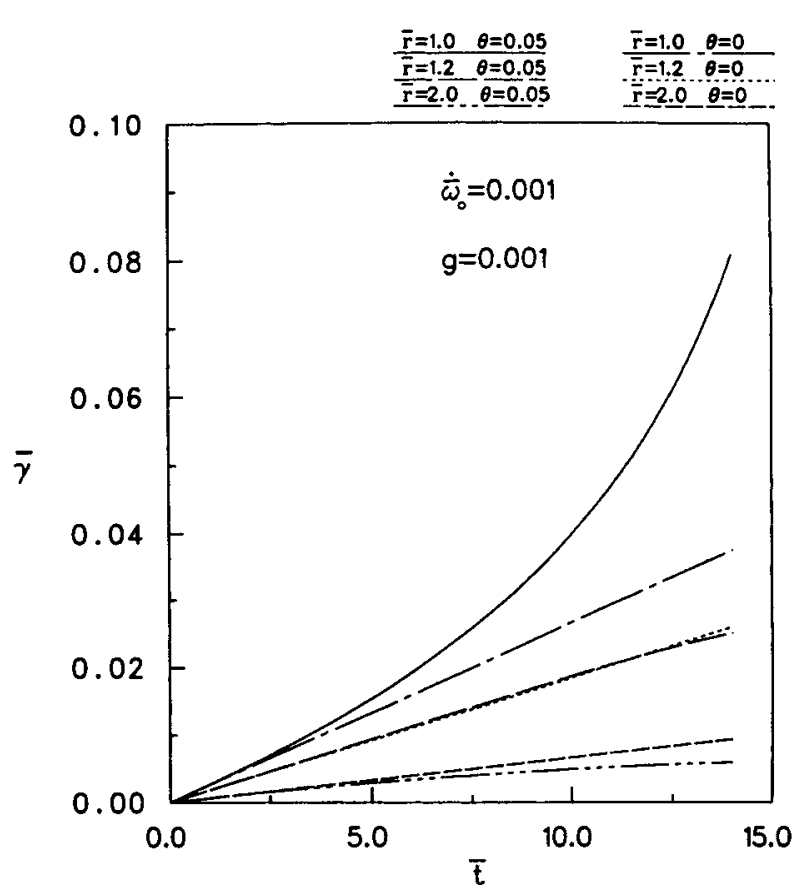

Fig. 10. Rotation control: shear strain us. time at radii $\bar{\tau}=1$ 1.2 , and 2.0 .

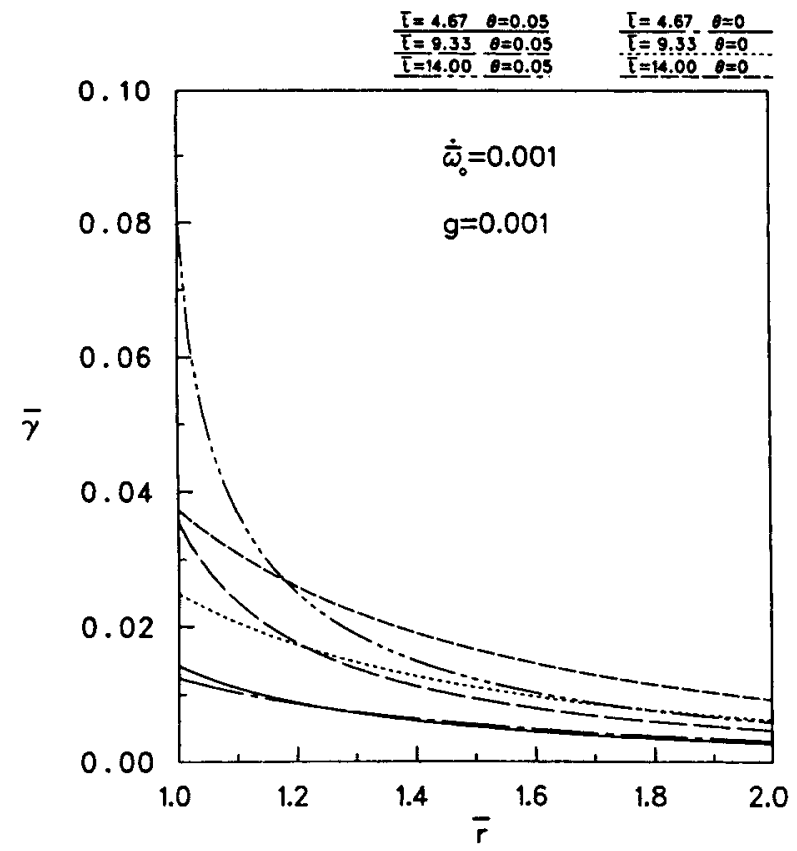

Fig. 11. Rotation control: shear strain us. radius for times $\bar{t}=4.67,9.33$, and 14.0.

growth of the strain histories and the rotation of the outer boundary.

These figures also show another important result. In the moment control case, a constant moment history means that each material element is subjected to a constant stress rate history. In the case of constant rotation rate for $\theta=0$, each material element undergoes a constant strain rate history. In contrast, a constant rotation rate for $\theta=0.05$ does not lead to constant shear strain rate histories at each material element. Figure 10 shows that the shear strain near the inner support grows faster than at a constant strain rate, while the shear strain in the outer portion grows slower than at a constant rate. In fact, no material element appears to undergo a shear strain history which corresponds to any of the standard histories discussed in the section entitled Yield.

The radial distribution of the strain, shown in Fig. 11 , plays a significant role in the interpretation of this behavior. For small time $\bar{t}$, the strains are small enough that they do not influence the relaxation process. According to Eq 22, the strains decay as $1 / \bar{r}^{2}$, so that the strains near the inner boundary are much greater than near the outer boundary. When the strains grow large enough near the inner boundary to accelerate stress relaxation, the strains near the outer boundary are still so small as to have negligible effect on stress relaxation. This is seen in Figs. 12 and 13 , which show histories and radial distributions of $\vec{\xi}$, respectively. Finally, in regard to Figs. 10, when the moment is reduced, the stresses at all particles are reduced. Near the inner boundary, stress relaxation is fast enough that the strains continue to increase. Near the outer boundary, stress relaxation is only slightly influenced by strains. When the stress decreases, so does the growth of strains.

Plots of shear stress $v$ s. shear strains are shown in Figs. 14 to 16 for material elements at $\bar{r}=1,1.2$, and 2 respectively. Note that each graph has a local maximum, which normally is associated with yield. However, the reason for this local maximum depends on the location of the material elements. For elements

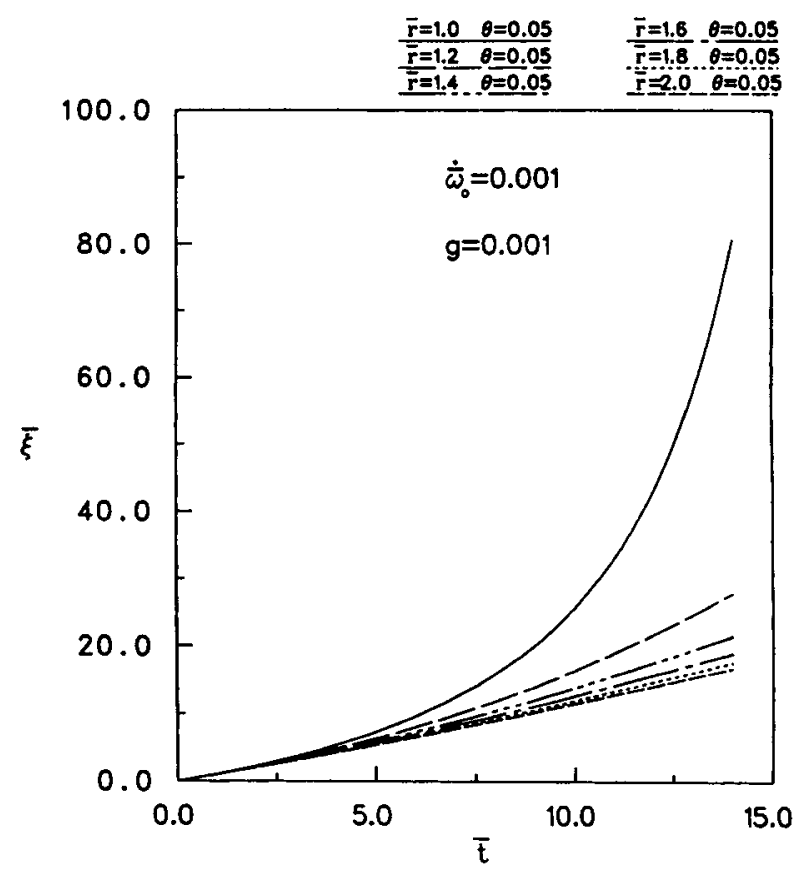

Fig. 12. Rotation control: reduced time us. time at several radii. 


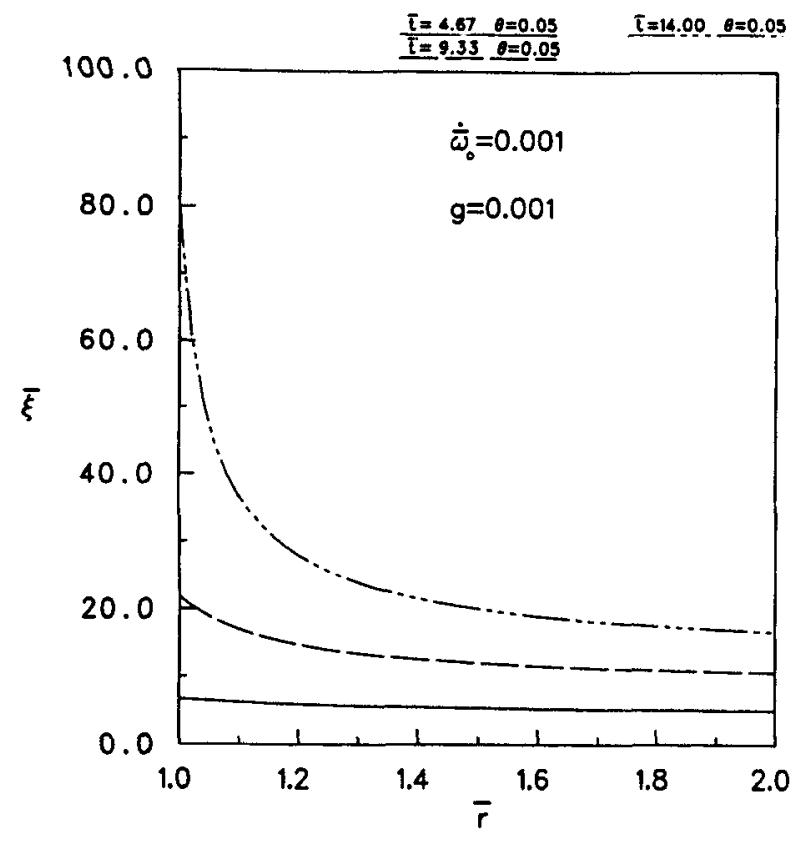

Fig. 13. Rotation control: reduced time us. radius for times $\bar{t}=4.67,9.33$, and 14.0 .

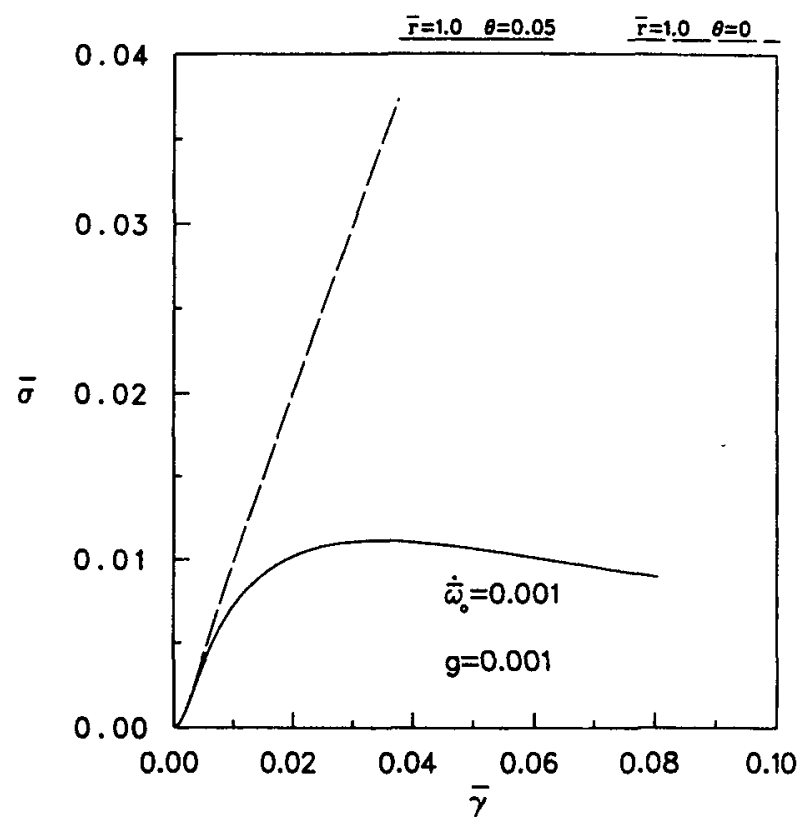

Fig. 14. Rotation control: shear stress vs. shear strain at radius $\bar{r}=1.0$.

near the inner boundary, the shear strain history grows sufficiently rapidly that the conditions for yield discussed previously are met. On the other hand, the shear strain in the elements in the outer layer becomes almost constant. The stress decreases there due to stress relaxation.

\section{ACKNOWLEDGMENT}

This work was supported in part by a grant from the National Science Foundation, No. DMR-8708405.

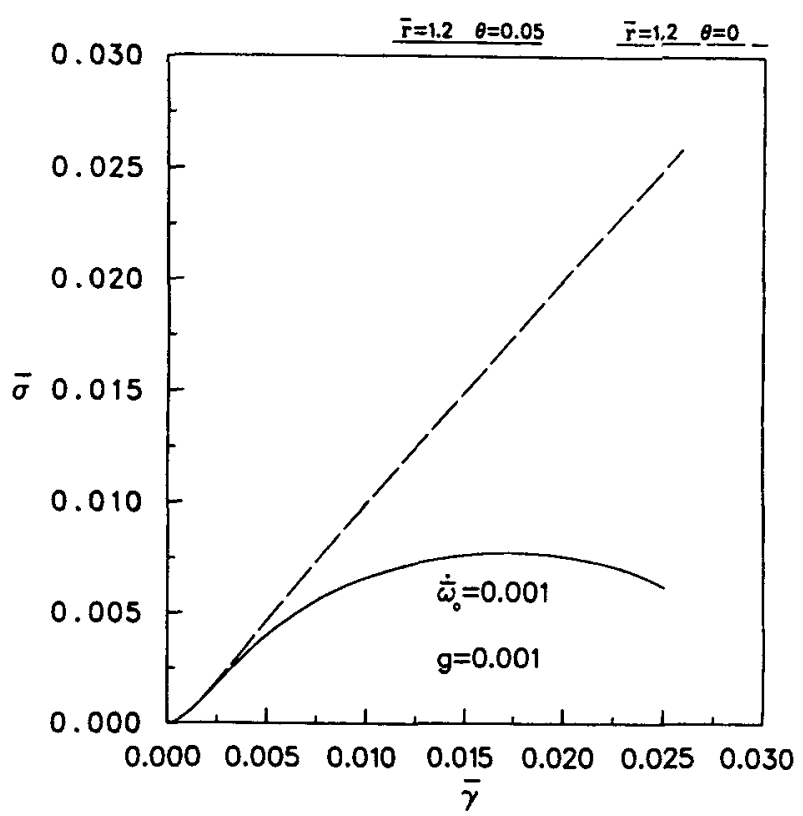

Fig. 15. Rotation control: shear stress vs. shear strain at radius $\bar{r}=1.2$.

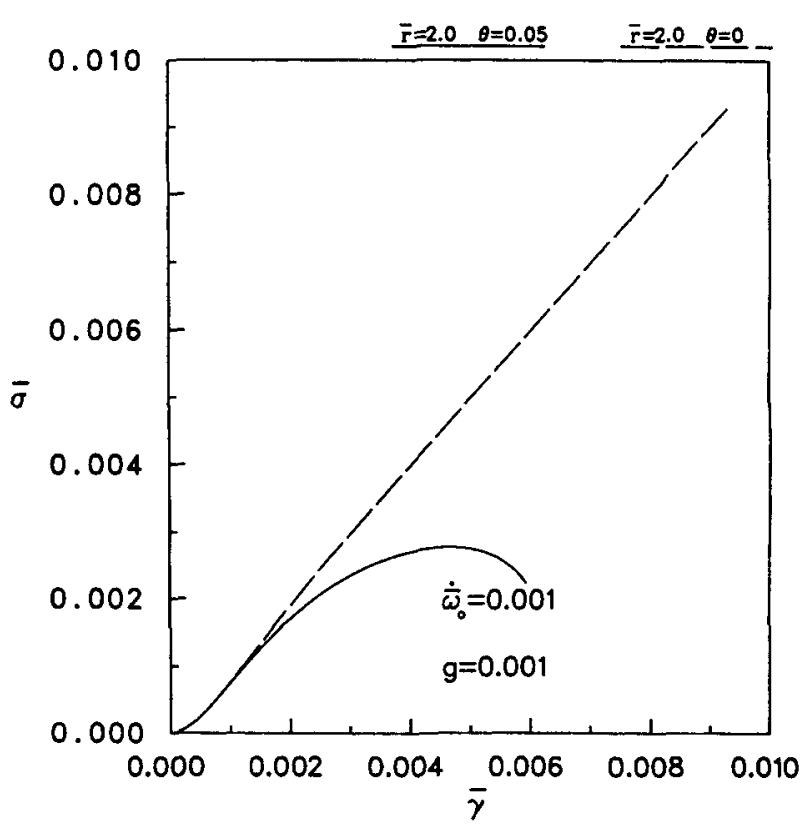

Fig. 16. Rotation control: shear stress us. shear strain at radius $\bar{r}=2.0$.

\section{REFERENCES}

1. R. M. Shay, Jr., and J. M. Caruthers, J. Rheol., 30, 781 (1986).

2. R. M. Shay, Jr., and J. M. Caruthers, Advances in Rheology, Proc. LXth Intl. Congress on Rheology, Mexico, 1, 549 (1984).

3. R. M. Shay, Jr., and J. M. Caruthers, Proc. 20th Midwestem Mechanics Conference, Purdue University, 14b. 493 (1987). 
A. S. Wineman and W. K. Waldron, Jr.

4. R. M. Shay, Jr., and J. M. Caruthers, Proc. Xth Intl. Congress on Rheology, Sydney, 2, 266 (1988).

5. W. G. Knauss and I. J. Emri, Comput. Struct., 13, 123 (1981).

6. W. G. Knauss and I. J. Emri, Polym. Eng. Sci., 27, 86 (1987).

7. P. B. Bowden, The Physics of Glassy Polymers, R. N. Haward, ed., Wiley, New York (1973).

8. L. M. Carapellucci and A. F. Yee, Polym. Eng. Sci., 26, 920 (1986).

9. A. F. Yee and P. D. DeTorres, Polym Eng. Sci, 14, 691 (1974).
10. R. E. Robertson, J. Chem. Phys., 44, 3950 (1966).

11. C. G'Sell and A. J. Gopez, J. Mater. Sci, 20, 3462 (1985).

12. G. B. McKenna and L. J. Zapas, J. Rheol., 23, 151 (1979).

13. I. M. Ward, Mechanical Properties of Solid Polymers, 2nd Ed., Wiley, New York (1983).

14. P. Linz. Analytical and Numerical Methods for Volterra Equations, Society of Industrial Engineering and Applied Mathematics, Philadelphia, (1985).

15. A. S. Wineman, J. Non-Newt. Fluid Mech., 4, 249 (1978). 